\title{
COMPARISON OF CORTISONE ACETATE AND A CODEINE MIXTURE AS ADJUVANT TREATMENT IN JUDET ARTHROPLASTY
}

BY

\author{
G. P. ARDEN \\ Heatherwood Orthopaedic Hospital
}

(RECEIVED FOR PUBLICATION FEBRUARY 27, 1956)

Experimental work by Ragan, Howes, and their associates $(1949,1950)$ has shown that fibrous scarring is decreased by cortisone. Hip arthroplasty may be followed by excessive fibrosis and by ectopic new bone formation which causes considerable disability. Cortisone given locally and systemically has been shown by Stinchfield (1950) to improve this condition in nylon hip arthroplasty in dogs. We have therefore investigated the use of cortisone as an adjuvant to Judet arthroplasty in man.

\section{Selection of Cases}

Eighteen patients (two bilateral=twenty hips) were selected, suffering from either degenerative changes of the hip or from rheumatoid arthritis with pain severe enough to justify operative treatment, conservative measures having failed. Cases were selected at random for treatment by cortisone or codeine by a system of numbered envelopes in series, only the hospital pharmacist knowing which patients were receiving cortisone.

The patients were admitted to the hospital one week before operation.

\section{Treatment}

Cortisone acetate was given in a pink, raspberryflavoured mixture in mist. codeine co. which alone was used for the control series. Cortisone was started 6 days before the operation at a dosage level of $150 \mathrm{mg}$./day in three divided doses for the first 4 days, then $100 \mathrm{mg}$. in four doses for the following 8 days, $75 \mathrm{mg}$. in three doses for 8 days, and finally $50 \mathrm{mg}$. for 6 days, making a total of $23 \mathrm{~g}$. cortisone acetate in 26 days.

The basic codeine mixture used for the control group contained aspirin gr. 4, phenacetin gr. 4, codeine phosphate gr. $\frac{1}{8}$, to the half ounce; it was given at the level of $1 \mathrm{oz}$. three times a day for 4 days, then $\frac{1}{2} \mathrm{oz}$. four times a day for 8 days, then $\frac{1}{2} \mathrm{oz}$. three times a day for 8 days, and finally $\frac{1}{2} \mathrm{oz}$. twice a day for 6 days, making a total of $11.5 \mathrm{gr}$. codeine in 26 days.

Those treating the patients did not know which cases were on codeine or cortisone (the severe oedema of legs in a patient on codeine was mistakenly attributed to cortisone).

\section{Methods of Assessment}

Pain.-This was assessed in four grades from $0-3$, and measured before operation, at weekly intervals until discharge, and then at 3,6 , and 12 months.

Range of Movement in Hip.-Clinical assessments of angular movement were taken without the use of a goniometer from a position of full extension of the hip. After the operation the range of movement of the affected hip was noted weekly until discharge, and then in the out-patients department after 3 months and 6 months.

Functional Result.-This was assessed 6 months after operation on the following basis:

(i) Good.-Able to walk one mile 3 months after operation. No real pain in hip. Has returned to former occupation or activity. Uses one stick only.

(ii) Fair._Able to walk up to half a mile. Some pain in hip. Has returned to light work or limited activity at home.

(iii) Poor.-Able to walk in house only. Considerable pain in hip. Unable to work and confined to house or bed. 
TABLE I

CASE ANALYSIS

\begin{tabular}{|c|c|c|c|c|c|c|c|c|c|c|c|c|c|c|}
\hline \multirow[b]{2}{*}{ Drug } & \multirow[b]{2}{*}{$\begin{array}{l}\text { Hip } \\
\text { No. }\end{array}$} & \multirow[b]{2}{*}{$\begin{array}{l}\text { Age } \\
\text { (yrs) }\end{array}$} & \multirow[b]{2}{*}{ Sex } & \multirow[b]{2}{*}{ Diagnosis } & \multirow{2}{*}{$\begin{array}{c}\text { Hip } \\
\text { In- } \\
\text { volved }\end{array}$} & \multirow{2}{*}{$\begin{array}{l}\text { Dura- } \\
\text { tion } \\
\text { of } \\
\text { Symp- } \\
\text { toms }\end{array}$} & \multicolumn{3}{|c|}{ Signs and Symptoms } & \multirow{2}{*}{$\begin{array}{l}\text { Tren- } \\
\text { delen- } \\
\text { burg's }\end{array}$} & \multirow{2}{*}{$\begin{array}{c}\text { Use } \\
\text { of } \\
\text { Sticks }\end{array}$} & \multicolumn{3}{|c|}{ Domestic Difficulties } \\
\hline & & & & & & & Pain & $\begin{array}{c}\text { Stiff- } \\
\text { ness }\end{array}$ & Limp & & & $\begin{array}{l}\text { Putting } \\
\text { on } \\
\text { Shoes }\end{array}$ & $\begin{array}{l}\text { Going } \\
\text { Up- } \\
\text { stairs }\end{array}$ & Bathing \\
\hline \multirow{6}{*}{ Cortisone } & $\left\{\begin{array}{l}1 \\
2\end{array}\right.$ & 59 & $\mathbf{F}$ & $\begin{array}{l}\text { Rheumatoid } \\
\text { Arthritis } \\
\text { Rheumatoid } \\
\text { Arthritis }\end{array}$ & $\begin{array}{l}\mathbf{L} \\
\mathbf{R}\end{array}$ & 12 yrs & \multirow{6}{*}{$7 / 7$} & \multirow{6}{*}{$2 / 7$} & \multirow{6}{*}{$3 / 7$} & \multirow{6}{*}{$4 / 7$} & \multirow{6}{*}{$4 / 7$} & \multirow{6}{*}{$6 / 6$} & \multirow{6}{*}{$0 / 6$} & \multirow{6}{*}{$2 / 6$} \\
\hline & 3 & 46 & $\mathbf{F}$ & $\begin{array}{l}\text { Osteo- } \\
\text { Arthritis }\end{array}$ & $\mathbf{R}$ & $1 \mathrm{yr}$ & & & & & & & & \\
\hline & 4 & 75 & $\mathbf{F}$ & $\begin{array}{l}\text { Osteo- } \\
\text { Arthritis }\end{array}$ & $\mathbf{R}$ & $1 \mathrm{yr}$ & & & & & & & & \\
\hline & 6 & 40 & $\mathbf{M}$ & $\begin{array}{c}\text { Osteo- } \\
\text { Arthritis }\end{array}$ & $\mathbf{L}$ & 14 mths & & & & & & & & \\
\hline & 9 & 61 & $\mathbf{F}$ & $\begin{array}{c}\text { Rheumatoid } \\
\text { Arthritis }\end{array}$ & $\mathbf{L}$ & 9 yrs & & & & & & & & \\
\hline & 10 & 68 & $\mathbf{F}$ & $\begin{array}{l}\text { Osteo- } \\
\text { Arthritis }\end{array}$ & $\mathbf{R}$ & $10 \mathrm{yrs}$ & & & & & & & & \\
\hline \multirow{7}{*}{ Codeine } & $\left\{\begin{array}{l}11 \\
12\end{array}\right.$ & 62 & $\mathbf{F}$ & $\begin{array}{l}\text { Rheumatoid } \\
\text { Arthritis } \\
\text { Rheumatoid } \\
\text { Arthritis }\end{array}$ & $\begin{array}{l}\mathbf{L} \\
\mathbf{R}\end{array}$ & $6 \mathrm{yrs}$ & \multirow{7}{*}{$8 / 8$} & \multirow{7}{*}{$2 / 8$} & \multirow{7}{*}{$8 / 8$} & \multirow{7}{*}{$5 / 8$} & \multirow{7}{*}{$5 / 8$} & ? & \multirow{7}{*}{$5 / 8$} & \multirow{7}{*}{$1 / 8$} \\
\hline & 13 & 63 & $\mathbf{F}$ & $\begin{array}{c}\text { Rheumatoid } \\
\text { Arthritis }\end{array}$ & $\mathbf{L}$ & $4 \mathrm{yrs}$ & & & & & & & & \\
\hline & 14 & 64 & $\mathbf{M}$ & $\begin{array}{l}\text { Osteo- } \\
\text { Arthritis }\end{array}$ & $\mathbf{R}$ & $8 \mathrm{mths}$ & & & & & & & & \\
\hline & 16 & 52 & $\mathbf{M}$ & $\begin{array}{l}\text { Osteo- } \\
\text { Arthritis }\end{array}$ & $\mathbf{R}$ & $6 \mathrm{mths}$ & & & & & & $7 / 8$ & & \\
\hline & 17 & 60 & $\mathbf{M}$ & $\begin{array}{l}\text { Osteo- } \\
\text { Arthritis }\end{array}$ & $\mathbf{R}$ & $1 \mathrm{yr}$ & & & & & & & & \\
\hline & 18 & 65 & $\mathbf{M}$ & $\begin{array}{l}\text { Osteo- } \\
\text { Arthritis }\end{array}$ & $\mathbf{R}$ & $10 \mathrm{mths}$ & & & & & & & & \\
\hline & 20 & 72 & $\mathbf{F}$ & $\begin{array}{l}\text { Osteo- } \\
\text { Arthritis }\end{array}$ & $\mathbf{L}$ & 2 yrs & & & & & & & & \\
\hline
\end{tabular}

\section{Results}

Of the eighteen patients taken into the trial, of whom one in each group had two hips involved, five cases had their treatment discontinued between the 8th and the 20th day of the trial on account of toxic effects (three in the cortisone group with oedema of the legs plus chest infection, plus venous thrombosis, urinary infection, and prostatic obstruction respectively, and two in the codeine group with coronary thrombosis and severe oedema of legs). This left 13 patients (15 hips) completing the trial: these two residual groups are comparable with those at the beginning of the trial (Table I).

Table II (opposite) shows that there was no real difference between the patients treated with cortisone and those treated with codeine. There was increase in movement up to 6 months, but this was not sustained in the group as a whole by the year. Pain, however, was improved, as was also the functional assessment.

\section{Summary}

(1) Two small comparable series of cases of hip disease, one treated with cortisone and the other with a codeine mixture before, during, and after a Judet arthroplasty, are discussed, and the results are compared.

(2) After 6 months and one year there was no difference between the two groups in regard to range of movement and degree of pain, and the final function was equal in both groups.

(3) No severe toxic effects were noted and there was no trouble with wound healing or wound infection.

My thanks are due to Dr. E. G. L. Bywaters for his encouragement and advice during the period of this trial and for his helpful criticisms in the preparation of this paper.

For the cortisone allocated for this trial I should like to thank the Medical Research Council and the Nuffield Foundation Joint Committee on Clinical Trials of 
TABLE II

POST-OPERATIVE RESULTS

\begin{tabular}{|c|c|c|c|c|c|c|c|c|c|c|}
\hline \multirow[b]{2}{*}{ Drug } & \multirow[b]{2}{*}{$\begin{array}{l}\text { Hip } \\
\text { No. }\end{array}$} & \multicolumn{2}{|c|}{ Pre-operative } & \multicolumn{2}{|c|}{ After 3 months } & \multicolumn{3}{|c|}{ After 6 months } & \multicolumn{2}{|c|}{ After 12 months } \\
\hline & & Flexion & Pain & $\begin{array}{c}\text { Post- } \\
\text { operative } \\
\text { Flexion }\end{array}$ & $\begin{array}{c}\text { Post- } \\
\text { operative } \\
\text { Pain }\end{array}$ & $\begin{array}{l}\text { Post- } \\
\text { operative } \\
\text { Flexion }\end{array}$ & $\begin{array}{c}\text { Post- } \\
\text { operative } \\
\text { Pain }\end{array}$ & $\begin{array}{c}\text { Distance } \\
\text { Walked } \\
(\mathrm{mls})\end{array}$ & $\begin{array}{c}\text { Post- } \\
\text { operative } \\
\text { Flexion }\end{array}$ & $\begin{array}{c}\text { Assess- } \\
\text { ment }\end{array}$ \\
\hline \multirow{8}{*}{ Cortisone } & $1 \mathrm{~L}$ & 50 & 3 & 70 & 0 & 70 & 1 & $\frac{1}{4}$ & 45 & Fair \\
\hline & $2 \mathbf{R}$ & 85 & 2 & 80 & 0 & 90 & 1 & $\frac{1}{4}$ & 60 & Fair \\
\hline & 3 & 80 & 3 & 90 & 2 & 80 & 2 & $\frac{1}{2}$ & 70 & Fair \\
\hline & 4 & 80 & 3 & 80 & 1 & 100 & 1 & $100 \mathrm{yds}$ & 20 & Fair \\
\hline & 6 & 90 & 3 & 80 & 1 & 100 & 0 & $\frac{1}{2}$ & 90 & Good \\
\hline & 9 & 30 & 3 & 75 & 0 & 70 & 1 & 1 & 80 & Fair \\
\hline & 10 & 75 & 3 & 80 & 0 & 100 & 0 & 1 & 80 & Good \\
\hline & Average & 70 & & 78 & & 87 & & & $63 \cdot 4$ & \\
\hline \multirow{9}{*}{ Codeine } & $11 \mathrm{~L}$ & 80 & 3 & 80 & 3 & 80 & 2 & $\frac{1}{2}$ & 80 & Fair \\
\hline & $12 \mathrm{R}$ & 60 & 3 & 75 & 0 & 90 & 1 & $\frac{1}{2}$ & 90 & Fair \\
\hline & 13 & 15 & 3 & 40 & 0 & 40 & 0 & $\begin{array}{l}\text { house- } \\
\text { bound }\end{array}$ & 25 & Fair \\
\hline & 14 & 40 & 3 & 90 & 1 & 100 & 0 & 3 & 100 & Fair \\
\hline & 16 & 80 & 2 & 90 & 0 & 100 & 0 & 2 & 90 & Good \\
\hline & 17 & 70 & 2 & 80 & 0 & 90 & 0 & $\frac{1}{2}$ & 90 & Good \\
\hline & 18 & 90 & 3 & 90 & 0 & 90 & 1 & 3 & 90 & Poor \\
\hline & 20 & 90 & 2 & 40 & 2 & 90 & 1 & $100 \mathrm{yds}$ & 30 & Good \\
\hline & Average & 66 & & 73 & & 85 & & & 74 & \\
\hline
\end{tabular}

Cortisone, ACTH, and Other Therapeutic Measures in the Chronic Rheumatic Diseases.

\section{REFERENCES}

Ragan, C., Howes, E. L., Plotz, C. M., Meyer, K., and Blunt, J. W. (1949). Proc. Soc. exp. Biol. (N.Y.), 72, 718. $-, \frac{1}{\text { Acad. Med., 26, }}, \frac{1}{251}$, and Lattes, R. (1950). Bull. N.Y. Stinchfield, F. E. (1950). J. Bone Jt Surg., 32-A, 739.

Comparaison de l'acétate de cortisone et d'une mixture de codéine comme traitement adjuvant à l'arthroplastie de Judet

\section{RÉSUMÉ}

(1) Deux petites séries semblables de cas de coxalgie l'une traitée à la cortisone et l'autre avec une mixture de codéine avant, pendant et après une arthroplastie de Judet, sont discutées et les résultats comparés.

(2) Après six mois et un an il n'y avait aucune différence entre les deux groupes en ce qui concerne l'amplitude de mouvement et l'intensité de la douleur et à la fin la fonction était égale dans les deux groupes.

(3) Aucun effet toxique sévère n'avait été constaté et il n'y avait eu aucun ennui au point de vue de la cicatrisation ou de l'infection.

Comparación del acetato de cortisona y de una mixtura de codeina como tratamineto adyuvante a la artroplastia de Judet

\section{SUMARIO}

(1) Se discuten y se comparan los resultados de dos pequeños grupos semejantes de casos de coxalgia, el uno tratado con la cortisona y el otro con una mixtura de codeina, antes, durante y después de una artroplastia de Judet.

(2) Después de seis meses y de un año no hubo diferencia alguna entre los dos grupos respecto a la amplitud del movimiento y la intensidad del dolor, y la función final fué igual en ambos grupos.

(3) No se notó ningún efecto tóxico grave y no hubo dificultades con cicatrización o con infección. 